\title{
Multiple Sclerosis in Children: Differential Diagnosis, Prognosis, and Disease-Modifying Treatment
}

\author{
Dejan Jakimovski ${ }^{1,2} \cdot$ Samreen Awan ${ }^{1} \cdot$ Svetlana P. Eckert $^{1} \cdot$ Osman Farooq $^{3,4} \cdot$ Bianca Weinstock-Guttman $^{1}$ (D)
}

Accepted: 2 December 2021 / Published online: 23 December 2021

(c) The Author(s), under exclusive licence to Springer Nature Switzerland AG 2021

\begin{abstract}
Pediatric-onset multiple sclerosis (POMS) is a rare neuroinflammatory and neurodegenerative disease that has a significant impact on long-term physical and cognitive patient outcomes. A small percentage of multiple sclerosis (MS) diagnoses occur before the age of 18 years. Before treatment initiation, a careful differential diagnosis and exclusion of other similar acquired demyelinating syndromes such as anti-aquaporin-4-associated neuromyelitis optica spectrum disorder (AQP4-NMOSD) and myelin oligodendrocyte glycoprotein antibody spectrum disorder (MOGSD) is warranted. The recent 2017 changes to the McDonald criteria can successfully predict up to $71 \%$ of MS diagnoses and have good specificity of $95 \%$ and sensitivity of $71 \%$. Additional measures such as the presence of T1-weighted hypointense lesions and/or contrast-enhancing lesions significantly increase the accuracy of diagnosis. In adults, early use of disease-modifying therapies (DMTs) is instrumental to a better long-term prognosis, including lower rates of relapse and disability worsening, and numerous FDA-approved therapies for adult-onset MS are available. However, unlike their adult counterparts, the development, testing, and regulatory approval of POMS treatments have been significantly slower and hindered by logistic and/or ethical considerations. Currently, only two MS DMTs (fingolimod and teriflunomide) have been tested in large phase III trials and approved by regulatory agencies for use in POMS. First-line therapies not approved by the FDA for use in children (interferon- $\beta$ and glatiramer acetate) are also commonly used and result in a significant reduction in inflammatory activity when compared with non-treated POMS patients. An increasing number of POMS patients are now treated with moderate efficacy therapies such as dimethyl fumarate and high-efficacy therapies such as natalizumab, anti-CD20 monoclonal antibodies, anti-CD52 monoclonal antibodies, and/ or autologous hematopoietic stem cell transplantation. These high-efficacy DMTs generally provide additional reduction in inflammatory activity when compared with the first-line medications (up to $62 \%$ of relapse-rate reduction). Therefore, a number of phase II and III trials are currently investigating their efficacy and safety in POMS patients. In this review, we discuss potential changes in the regulatory approval process for POMS patients that are recommended for DMTs already approved for the adult MS population, including smaller sample size for pharmacokinetic/pharmacodynamic studies, MRIcentered primary outcomes, and/or inclusion of teenagers in the adult trials.
\end{abstract}

\section{Introduction}

Bianca Weinstock-Guttman

bw8@buffalo.edu

1 Department of Neurology, Jacobs Comprehensive MS Treatment and Research Center, Jacobs School of Medicine and Biomedical Science, University of Buffalo, 1010 Main Street, Buffalo, NY 14202, USA

2 Department of Neurology, Buffalo Neuroimaging Analysis Center, Jacobs School of Medicine and Biomedical Sciences, University at Buffalo, State University of New York, Buffalo, NY, USA

3 Division of Pediatric Neurology, Oishei Children's Hospital of Buffalo, Buffalo, NY, USA

4 Department of Neurology, Jacobs School of Medicine, State University of New York at Buffalo, Buffalo, NY, USA
Multiple sclerosis (MS) is a chronic, neuroinflammatory and neurodegenerative disease of the central nervous system (CNS) that commonly affects the young adult population, between the ages of 20 and 50 years. In a small percentage of MS cases (ranging from 2 to 5\% based on different reports), the first demyelinating clinical attack can occur prior to the age of 18 years [1]. When compared with their adult counterparts, pediatric-onset MS (POMS) patients typically have a more inflammatory-active disease course, resulting in more frequent relapses, but slower long-term disability accumulation [2]. These features are generally attributed to the extensive post-relapse recovery that can be attributed to higher 


\section{Key Points}

There is a significant disparity in the availability of disease-modifying treatment (DMT) options between adult-onset multiple sclerosis (AOMS) and pediatriconset multiple sclerosis (POMS).

Only two MS-based medications were recently investigated in a double-blind, randomized, phase III POMS trial and received regulatory approval.

Multiple logistical and ethical concerns hinder the development and testing of DMTs for the POMS population.

ability for myelin repair/synthesis and greater plasticity of the developing brain [3].

Although POMS patients have relatively slower physical disability progression, the early and frequent neuroinflammatory attacks can result in impaired brain development and poorer cognitive performance when compared with adultonset MS (AOMS) patients or non-MS peers $[4,5]$. These impairments can have long-term consequences, including a lower likelihood of pursuing higher education, lower annual earning, frequent sick days during work life, and early enrollment into disability pension programs [6]. Therefore, efforts towards early diagnosis, discovery of early predictors of long-term outcomes, and appropriate early drug intervention are highly warranted [7].

This narrative review will focus on the current diagnosis and management of POMS, exploring the efficacy and safety characteristics of the currently available drug armamentarium. A succinct description of POMS diagnosis, differential diagnosis, and factors identified to interfere with MS long-term clinical outcomes will be presented also. Data reported in the manuscript was collected through a search of PubMed, EMBASE, and Scopus entries up to June 26, 2021 using MeSH terms such 'pediatric MS,' 'POMS,' 'multiple sclerosis' AND 'children' and 'DMT' OR 'disease-modifying treatment' AND 'Pediatric MS'. Additional literature derived from the references of the retrieved manuscripts and personal databases were utilized.

\section{Incidence and Prevalence of Pediatric-Onset Multiple Sclerosis (POMS)}

Comparably to the adult MS distribution, a systematic review performed in 2016 suggested significant geographical heterogeneity in the incidence and prevalence of POMS
[8]. Data ranged from the lowest incidence of 0.05 per 100,000 children in Tunisia to 2.85 per 100,000 children in Sardinia, Italy [8]. In addition to the study from Sardinia showing higher incidence and one more from Kuwait (2.1 per 100,000), all remaining studies report incidence that was $<1$ case per 100,000 children [8]. A smaller number of studies also provided the overall prevalence of POMS, which ranges from 0.69 per 100,000 children in Japan to 26.92 cases per 100,000 children in Sardinia, Italy [8]. The latest update of the MS Atlas estimates that currently there are at least 30,000 children living with MS (data derived from 47 reporting countries).

A recent meta-analysis that included 13 epidemiological studies estimated the global annual incidence of pediatric onset MS at 0.87 per 100,000 individuals [9]. Regional subgroup analysis was only feasible for Europe $(0.78$ per 100,000 individuals) and the Middle East $(0.89$ per 100,000 individuals) [9]. Interestingly, the overall incidence differed based on which diagnostic criteria were used, with 0.92 per 100,000 individuals for the 2013 International Pediatric Multiple Sclerosis Study Group (IPMSSG) criteria and 1.67 per 100,000 individuals for the 2010 McDonald criteria [9]. The global prevalence of POMS cases was estimated at 8.11 cases per 100,000 people [9]. In comparison with the 2013 IPMSSG criteria and/or $2010 \mathrm{McDonald}$ criteria, another study that estimated the incidence and prevalence of POMS in Ontario, Canada utilized different diagnostic criteria that are based on administrative data claims [10]. Based on their findings, this world region had the highest POMS burden with a recent increase in annual POMS incidence, going from 0.61 per 100,000 in 2003 to 0.98 or 1.24 per 100,000 in 2014 [10]. Based on their criteria, the study estimated that the Ontario region (with a population of over 14 million) had between 114 and 191 children living with MS [10].

In comparison, fewer studies have investigated the incidence and prevalence of pediatric aquaporin- 4 antibody-associated neuromyelitis optica spectrum disorder (AQP4-NMOSD). Between 2008 and 2018, a Danish population-based study recorded four such patients and estimated the incidence at 0.031 per 100,000 [11]. Similar incidence data was seen in Catalonia with pediatric NMOSD estimated at 0.037 per 100,000 [12]. The prevalence of pediatric NMOSD in Japan was estimated at 0.06 per 100,000 children [13]. With the upcoming development of myelin oligodendrocyte glycoprotein antibody spectrum disorder (MOGSD) diagnostic criteria and greater availability of antiMOG antibody testing, future studies will be able to better determine the incidence and prevalence of this neuroinflammatory entity and separate it from POMS. 


\section{Diagnosis of POMS and Differential Diagnosis}

An event of acquired CNS demyelination in children younger than 18 years old, also known as acquired demyelinating syndrome (ADS), could represent one of many neuroinflammatory diseases including AQP4-NMOSD, MOGSD, acute disseminated encephalomyelitis (ADEM) with encephalopathy, or a monophasic disease [14]. In fact, only $20 \%$ of pediatric ADS cases are ultimately diagnosed with POMS [15]. Table 1 demonstrates some of the key clinical, laboratory, and neuroimaging characteristics that can aid the differential diagnosis between the ADS presentations. Similar and more comprehensive illustrations can be found elsewhere. Moreover, the typical magnetic resonance imaging (MRI) findings seen in a 10-year-old MS patient, an 8-year-old AQP-4-positive NMOSD patient, an 11-year-old MOGSD patient, and an ADEM patient are shown in Fig. 1.

The diagnosis of POMS is undertaken through the lens of the adult McDonald MS diagnostic criteria and its latest 2017 revision. The diagnostic utility was recently analyzed in a nationwide study of 324 patients with ADS who were followed for 6 years after their first attack [15]. Over the follow-up period, a total of 71 (22\%) children were diagnosed with MS. The 2017 McDonald criteria successfully predicted $71 \%$ of the MS diagnoses with specificity and sensitivity of $95 \%$ and $71 \%$, respectively [15]. When compared with the 2010 revision (53\% correctly identified), the 2017 McDonald criteria contributed an additional 18\% increase in diagnostic sensitivity [15]. Apart from the McDonald criteria parameters, a random forest analysis isolated several differentiating features between multiple sclerosis and other ADS-based disorders, including the presence of at least one T1-weighted hypointense lesion (black hole), at least one gadolinium-enhancing lesion, at least one periventricular lesion, and presence of oligoclonal bands [15]. The use of these criteria had excellent sensitivity and specificity of $84 \%$ and $93 \%$, respectively [16]. The diagnosis of pediatric AQP4-positive or negative NMOSD should be performed through the recent adult diagnostic criteria, while formal MOGSD diagnostic criteria are currently in development [17]. Similar diagnostic algorithms that utilize a mixture of clinical, imaging, and serological features have been previously proposed and generally categorize patients in four main distinct phenotypes of MS, NMOSD with AQP4-Ab, MOG-Ab-associated disease, and seronegative relapsing demyelinating syndromes [18].

Table 1 Demographic, clinical, laboratory and imaging characteristics of the different acquired demyelinating syndrome presentations

\begin{tabular}{|c|c|c|c|}
\hline & POMS & AQP4-NMOSD & MOGSD \\
\hline $\mathrm{F}: \mathrm{M}$ & $2: 1$ & $4: 1$ & $1: 1$ \\
\hline Typical age of onset & $>10$ years old & $>10$ years old & $<10$ years old \\
\hline Monophasic or recurrent & Recurrent & Recurrent & $\begin{array}{l}50 \% \text { of MOGSD patients have } \\
\text { relapses and } 50 \% \text { have monopha- } \\
\text { sic disease }\end{array}$ \\
\hline \multicolumn{4}{|c|}{ Clinical and imaging features } \\
\hline Optic neuritis & $\begin{array}{l}\text { Unilateral short and focal ON } \\
\text { lesions }\end{array}$ & $\begin{array}{l}\text { Bilateral ON lesions involving the } \\
\text { chiasm and optic tracts }\end{array}$ & $\begin{array}{l}\text { More often having bilateral long } \\
\text { ON lesions when compared with } \\
\text { MS (unilateral ON can occur in } \\
\text { MOGSD) }\end{array}$ \\
\hline Papillitis & Rare & Rare & Common \\
\hline Transverse myelitis & $\begin{array}{l}\text { Short segments commonly affect- } \\
\text { ing C-spine }\end{array}$ & $\begin{array}{l}\text { Common LETM extending in } \\
\text { brainstem }\end{array}$ & Common LETM \\
\hline Infratentorial pathology & Small focal lesions & $\begin{array}{l}\text { Large cerebellar lesions extending } \\
\text { through the cerebellar peduncles } \\
\text { and mid-brain }\end{array}$ & $\begin{array}{l}\text { Large cerebellar lesions extending } \\
\text { through the cerebellar peduncles } \\
\text { can occur more often than in MS }\end{array}$ \\
\hline ADEM presentation & Rare & Occasional & Common initial presentation \\
\hline \multicolumn{4}{|l|}{ CSF analysis } \\
\hline Anti-AQP4 & Never present & Always present & Never present \\
\hline Anti-MOG & Very rare & Never present & Always present \\
\hline OCB & Common & Rare & Rare \\
\hline Pleocytosis & Frequent & Common & Common \\
\hline
\end{tabular}

Table modified from Fadda et al. [14]

$A D E M$ acute disseminated encephalomyelitis, AQP4-NMOSD aquaporin-4 neuromyelitis optica spectrum disorder, CSF cerebrospinal fluid, $F: M$ female:male, LETM longitudinal extensive transverse myelitis, MOGSD myelin oligodendrocyte glycoprotein antibody spectrum disorder, $M S$ multiple sclerosis, $O C B$ oligoclonal bands, $O N$ optic neuritis, $P O M S$ pediatric-onset multiple sclerosis 
Fig. 1 MRI-based differential diagnosis of pediatric patients with multiple sclerosis, neuromyelitis optica spectrum disorder, myelin oligodendrocyte spectrum disorder, and acute demyelinating encephalomyelitis. A, B Axial FLAIR images demonstrating typical MS-based hyperintensities within the periventricular region, juxtacortical region, and in the posterior fossa (cerebellum). C, D Sagittal T2 image of the spinal cord and axial FLAIR image demonstrating longitudinal extensive transverse myelitis that involves the brainstem region and extend over the entire cervical portion of the spinal cord. E, F Axial post-contrast FLAIR image demonstrating bilateral optic neuritis with optic nerve edema associated with contrast enhancement of the optic nerve and perineural sheet. Coronal T2 image demonstrating cervical spinal cord lesion spanning over 2.5 vertebral levels. G, H Axial FLAIR images demonstrate multiple, diffuse, 'fluffy,' poorly defined $\mathrm{T} 2$ hyperintense lesions involving both grey and white matter and both supra- and infratentorial region. ADEM acute disseminated encephalomyelitis, FLAIR fluid-attenuated inversion recovery MOGSD myelin oligodendrocyte glycoprotein antibody spectrum disorder, $M S$ multiple sclerosis, NMOSD neuromyelitis optica spectrum disorders

A recent European Union (EU)-based pediatric MOG consortium consensus was provided, with multiple separate statements addressing the MOG-associated clinical features [19], neuroimaging features [20], the use of different available assays for MOG-antibody testing and other biomarkers such as neurofilament light chain [21], the clinical outcomes of pediatric MOGSD [22], and a final part that reviews the current acute and maintenance treatment options [23].

\section{Disability Outcomes, Predictors, and Importance of Treatment in POMS}

The earliest large data reporting on long-term outcome in POMS came from the European Database for Multiple Sclerosis (EDMUS) network, which included 394 patients $\leq 16$ years old with the diagnosis of MS (7.6\% of patients with diagnosis were $<10$ years old). These data suggested that the transition into secondary progressive MS occurred after a median of 28.1 years and at a median age of 41.4 years [2]. Moreover, the POMS patients had disability progression to an Expanded Disability Status Scale (EDSS) score of 6.0 (not able to walk for $>100 \mathrm{~m}$ without the need of unilateral support) after a median disease duration of 28.9 years [2]. Although the time from disease onset to certain EDSS scores was significantly longer when compared with their AOMS counterparts, POMS patients were still on average 10 years younger [2]. Approximately 50\% of these POMS patients were at some point treated with disease-modifying therapy (DMT), but most data stemmed from early 1976 to 2001 when none of the patients received treatment early in their disease course. To better account for the disease duration as part of the disease severity, a recent adaptation
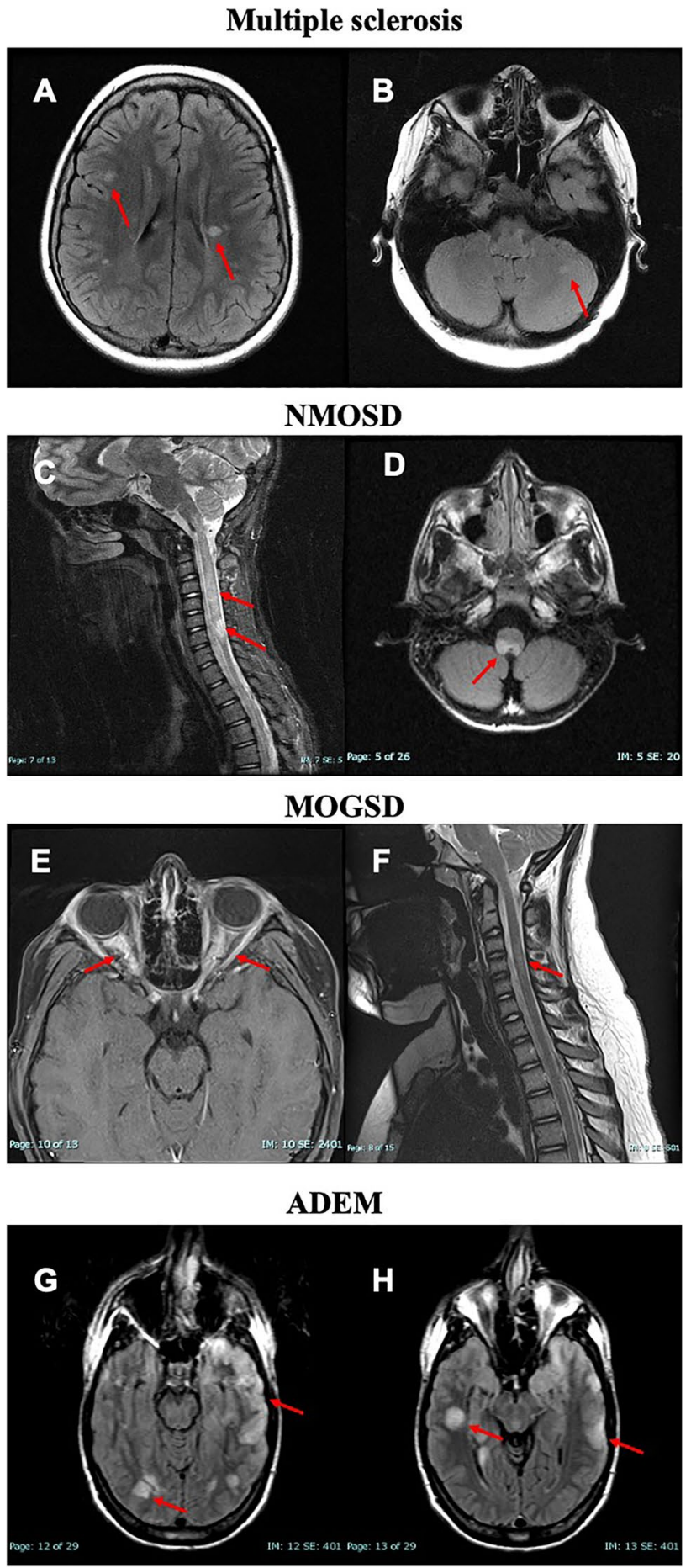

of the Multiple Sclerosis Severity Scale (MSSS) for POMS patients has been proposed (Ped-MSSS) [24].

Over the last several years, multiple large observational studies have reported on long-term outcomes in patients with POMS [7, 25]. A recent Italian registry study included a total of 3198 patients with POMS who were followed for an average of 21.8 years [7]. The time to reach different disability levels was significantly decreased with each 
diagnosis epoch (up to 50-70\% when compared with the natural history), which can be attributed mainly to the initiation of highly efficacious drugs during the pediatric age and continued use of DMT for a longer period of time when compared with prior epochs [7]. In a similar fashion, 549 POMS patients from the Swedish MS registry also took significantly longer time to reach certain EDSS milestones, but still were younger when compared with the AOMS cohort [25]. Faster progression in this POMS group was associated with a greater number of relapses during the first 5 years of the disease (5.35 times greater risk of reaching EDSS 4.0) and being classified with a primary progressive (PP) course (4.63 times greater risk), while complete recovery from the first relapse was associated with 59\% lower risk of reaching an EDSS of 4.0 [25].

Reports also suggest additional predictors of long-term outcomes, including the occurrence of the first two demyelinating attacks within the same year of disease onset as being associated with a greater risk of new relapse and progression to EDSS disability score of 4.0 [26]. Moreover, POMS patients with the first demyelinating attack affecting the brainstem have a significantly greater risk for future disability progression (up to 5 times) when compared with patients with lesions in other CNS locations [27]. In a recent 9-year follow-up study, early treatment with high-efficacy medication resulted in a significantly longer time to a new relapse [28]. The presence of cerebellar lesions at baseline and high-efficacy medications was also associated with a lower long-term annualized relapse rate (ARR), whereas presentation of transverse myelitis was associated with a higher ARR over the follow-up [28]. In terms of long-term disability outcome, the occurrence of two new T2 hyperintensities and an EDSS increase within the first 2 years of the disease were able to predict 9-year disability worsening [28].

The treatment effect was also seen in the Danish MS Registry, which also showed that delay of MS treatment for 2 years is associated with approximately two additional relapses over the next 3 years when compared with patients that started early treatment [29]. Moreover, late starters had a slightly higher risk for future confirmed disability worsening [29]. In particular, delay of treatment for 2 years resulted in 2.52 times greater risk of reaching an EDSS score of 4.0, and every year of DMT delay was further associated with a 17\% greater risk of reaching an EDSS score of 4.0 [30].

In addition to physical worsening, the POMS patients are also at risk of faster cognitive decline, which leads to earlier diagnosis of cognitive impairment when compared with their AOMS peers. Data from the aforementioned Swedish MS registry confirmed that POMS patients had significantly slower information processing speed (measured through the symbol digit modalities test; SDMT) and faster decline when compared with their AOMS peers [31]. These findings were independent of all demographic and clinical factors, including disease duration, type of disease course, and exposure to DMTs [31]. These changes can be attributed to inflammation-induced impairment in the maturation of the cortical and deep gray structures [32].

As with AOMS, factors such as male gender, non-Caucasian descent, infection with Epstein-Barr virus (EBV), higher body mass index (BMI), lower vitamin D levels, human leukocyte antigen (HLA)-DRB1*15:01, frequent childhood infections, and other modifiable risk factors are all associated with greater susceptibility and worse clinical outcomes in POMS patients [33-38].

\section{Use of Disease-Modifying Treatment in Pediatric Multiple Sclerosis}

The extensive inflammatory activity and fast accumulation of white and gray matter pathology within POMS patients necessitates early and often aggressive anti-inflammatory intervention. In past decades, the off-label pharmacological treatment of POMS patients was highly heterogeneous and subject to the personal preferences of the pediatric MS care providers. Although this approach resulted in useful information acquired through many open-label, observational POMS studies, the International Pediatric MS group still emphasizes the general lack of properly randomized trials and regulatory approval for DMT in POMS. Hereafter, we describe the efficacy and safety data of each MS medication that has been used in the POMS population, which is either derived from real-world retrospective and observational registries or a few randomized interventional studies (Table 2). For further clarity, we review the medications in the order of their original approval.

\subsection{Interferon- $\beta$ and Glatiramer Acetate}

Interferon- $\beta$ and glatiramer acetate have been utilized as first-line medications in POMS ever since their availability in the MS armamentarium for AOMS. Although the exact mechanism of action of interferon- $\beta$ in MS is not fully understood, its beneficial effects are associated with downregulation of the major histocompatibility complex (MHC) class II, induction of interleukin-10 production, a shift of the T-cell balance towards the anti-inflammatory T-helper 2 cells, and decrease in matrix metalloproteinases (MMPs) [39]. Glatiramer acetate is composed of multiple synthetic copolymers of amino acids that are intended to mimic the myelin basic protein (MBP) and act as a T-cell receptor antagonist [40]. This leads to a similar shift in cytokines and T-cell response towards a greater anti-inflammatory profile [40]. In the early 2000s, several smaller open-label studies were performed for each of the available preparations, including subcutaneous (SC) interferon- $\beta$ - $1 \mathrm{~b}\left(\right.$ Betaseron $^{\circledR}$ ), 
Table 2 Current studies investigating disease-modifying treatments in pediatric MS patients that are registered in ClinicalTrials.gov

\begin{tabular}{|c|c|c|c|c|}
\hline Medication & Design & No. of patients & $\begin{array}{l}\text { Main efficacy findings on relapse } \\
\text { rate }\end{array}$ & Clinical trial \\
\hline $\begin{array}{l}\text { Subcutaneous interferon- } \beta-1 \mathrm{a} \\
\text { (REPLAY) }\end{array}$ & Retrospective observational & 307 & $\begin{array}{l}\text { Significant ARR reduction com- } \\
\text { pared with prior to drug initiation } \\
(1.79-0.47)\end{array}$ & NCT01207648 \\
\hline Interferon $\beta-1 \mathrm{~b}$ (BETAPAEDIC) & $\begin{array}{l}\text { Prospective, open-label, observa- } \\
\text { tional }\end{array}$ & 68 & $\begin{array}{l}\text { Significant ARR reduction com- } \\
\text { pared with prior to drug initiation } \\
(2.2-1.0)\end{array}$ & NCT00963833 \\
\hline Peginterferon $\beta$-1a & $\begin{array}{l}\text { Open-label, randomized, active- } \\
\text { controlled }\end{array}$ & 142 & Currently ongoing & NCT03958877 \\
\hline Fingolimod (PARADIGMS) & $\begin{array}{l}\text { Double-blind, randomized, active- } \\
\text { comparator }\end{array}$ & 215 & $\begin{array}{l}\text { Significant } 82 \% \text { decrease in the } \\
\text { ARR when compared with IM } \\
\text { interferon } \beta-1 \mathrm{a}\end{array}$ & NCT01892722 \\
\hline Dimethyl fumarate (FOCUS) & $\begin{array}{l}\text { 24-week, phase II, single-arm, } \\
\text { open-label }\end{array}$ & 20 & $\begin{array}{l}\text { Reduced ARR from } 1.5 \text { for the year } \\
\text { before entry into the study to } 0.8 \\
\text { during the } 24 \text {-week study period }\end{array}$ & NCT02410200 \\
\hline $\begin{array}{l}\text { Dimethyl fumarate (CON- } \\
\text { NECTED) }\end{array}$ & $\begin{array}{l}\text { 96-week follow-up for the FOCUS } \\
\text { study }\end{array}$ & 20 & $\begin{array}{l}\text { ARR remained low at } 0.2 \text {, an } \\
84.5 \% \text { reduction when compared } \\
\text { with the year before entry into } \\
\text { the study }\end{array}$ & {$[75]$} \\
\hline Dimethyl fumarate (CONNECT) & $\begin{array}{l}\text { Open-label, randomized, active- } \\
\text { comparator }\end{array}$ & 156 & Currently ongoing & NCT02283853 \\
\hline $\begin{array}{l}\text { Dimethyl fumarate and peginter- } \\
\text { feron } \beta-1 \mathrm{a}\end{array}$ & $\begin{array}{l}\text { 3-arm, double-blind, placebo-con- } \\
\text { trolled, randomized }\end{array}$ & 260 & Currently ongoing & NCT0380763 \\
\hline Teriflunomide (TERIKIDS) & $\begin{array}{l}\text { Double-blind, randomized, } \\
\text { placebo-controlled }\end{array}$ & 166 & $\begin{array}{l}\text { A non-significant } 34 \% \text { reduction } \\
\text { in risk of relapse when compared } \\
\text { with placebo }\end{array}$ & NCT02201108 \\
\hline Natalizumab & Open-label, PK/PD study & 13 & Completed, no results posted & NCT01884935 \\
\hline Natalizumab & Retrospective observational & 400 & Completed, no results posted & NCT02137109 \\
\hline Alemtuzumab (LemKids) & Open-label, non-randomized & 50 & Currently ongoing & NCT03368664 \\
\hline Ocrelizumab & Open-label, PK/PD study & 36 & Currently ongoing & NCT04075266 \\
\hline $\begin{array}{l}\text { Ofatumumab and siponimod } \\
\text { (NEOS) }\end{array}$ & $\begin{array}{l}\text { 3-arm double-blind, non-inferiority } \\
\text { randomized }\end{array}$ & 180 & Currently ongoing & NCT04926818 \\
\hline TCR peptide vaccine & Double-blind, randomized & 12 & Currently ongoing & NCT02200718 \\
\hline
\end{tabular}

$A R R$ annualized relapse rate, $I M$ intramuscular, $M S$ multiple sclerosis, $P K / P D$ pharmacokinetics/pharmacodynamics, $T C R$ T-cell receptor

intramuscular (IM) interferon- $\beta$-1a $\left(\right.$ Avonex $\left.^{\circledR}\right)$, and SC interferon- $\beta-1 \mathrm{a}\left(\right.$ Rebif $\left.^{\circledR}\right)$.

One of these was a retrospective observational study investigating the effectiveness of a smaller $22-\mu \mathrm{g}$ dose for SC interferon- $\beta$-1a $\left(\right.$ Rebif $\left.^{\circledR}\right)$ in 51 pediatric patients [41]. Due to continuous disease activity, almost half of the patients (22 out of 46) had to switch to the higher 44- $\mu$ g dose [41]. In spite of this, the interferon- $\beta$-1a treatment overall resulted in a decrease in ARR from 1.8 in the year before treatment to 0.8 during the 2-year study and the EDSS scores remained stable in 48 out of 51 patients [41]. A similarly structured study also investigated the effectiveness of SC interferon- $\beta-1 \mathrm{~b}$ (Betaseron ${ }^{\circledR}$ ), with a total of 42 pediatric patients being enrolled to either a full $250 \mu \mathrm{g}$ every other day or to a dosing regimen that uses quarterly escalation of the medication [42]. In the analysis of 38 patients with confirmed MS, interferon- $\beta-1 \mathrm{~b}$ resulted in a $50 \%$ reduction in the ARR [42]. Over the study period, 25 children discontinued their treatment, most commonly due to new disease activity and/or switching to other DMTs (reasons including perceived lack of efficacy, lack of adherence or funding, loss to follow-up, and adverse events such as injection pain) [42]. The treatment was associated with adverse events that were similar to the adult population, with elevation of liver function tests (ranging from 2 to 14 times above the upper limit of normal) in eight treated children, and occurrence of transient, mild leukopenia seen in two additional children [42]. Lastly, the effectiveness of IM interferon- $\beta$-1a (Avonex ${ }^{\circledR}$ ) was investigated in 52 POMS patients where it significantly decreased the ARR from 1.9 before treatment to 0.4 after approximately 2 years of treatment [43]. Similar to the other formulations, the adverse events were comparable to those seen in the adult population with flu-like syndrome, headache, myalgia, and fever being the most common ones 
[43]. Other adverse events included laboratory abnormalities such as leukopenia in six patients, three patients with transient reduction in free T3/T4, two patients with a transient increase in alanine transaminase (ALT)/aspartate aminotransferase (AST), and two more patients with transient increase in antinuclear antibody (ANA) titer [43].

After the aforementioned early studies, a set of larger observational studies provided more data regarding the efficacy and safety of the interferon- $\beta$ medications that were investigated either separately or in a bundled analysis that includes a mix of all interferon- $\beta$ preparations. As seen in the BENEFIT (Betaferon/Betaseron in Newly Emerging MS for Initial Treatment) [44], ETOMS (Early Treatment of Multiple Sclerosis Study) [45] and CHAMPS (Controlled HighRisk Avonex Multiple Sclerosis Study) [46] adult trials, the early administration of interferon- $\beta$ in pediatric patients with a first clinical attack (CIS) can significantly prolong the time to the next relapse and conversion to a relapsing-remitting (RR) form of the disease [47]. When compared with placebo, interferon- $\beta$ provides a $69 \%$ reduction in having a new attack during the first year of follow-up and a $60 \%$ reduction in the first 2 years [47]. The observational REPLAY (Retrospective Cohort Study of Rebif ${ }^{\circledR}$ Use in Pediatric MS Subjects) study included up to 307 POMS patients that started treatment with SC interferon- $\beta-1 \mathrm{a}$ and were followed for a median of 3.7 years [48]. As seen with the previous studies, the treatment resulted in a significant reduction of the ARR from 1.79 prior to drug initiation to 0.47 afterward [48]. Over the follow-up period, up to 99 patients discontinued their treatment due to a new clinical relapse $(n=31)$, new MRI-based disease activity $(n=9)$, other medical events $(n=44)$, or a personal decision $(n=15)$ [48]. Even in patients that stopped or switched their SC interferon- $\beta-1 \mathrm{a}$ treatment, the relapse rate remained low after the cessation of the treatment (no rebound of activity was recorded) [48]. Interestingly, the study also demonstrated some geographical differences, where despite the same relapse rate before treatment initiation, the effectiveness of SC interferon- $\beta-1 \mathrm{a}$ was smaller in the United States (US)-based POMS patients [49]. These differences could be attributed to the higher body mass index and greater rate of drug discontinuation/ switching in US POMS patients [49]. The BETAPAEDIC study (Study evaluating Betaferon ${ }^{\circledR}$ 's safety and tolerability in paediatric patients with multiple sclerosis) investigated the effectiveness of SC interferon- $\beta$ - $1 \mathrm{~b}$ in 67 treatment-naïve POMS patients that were followed for 2 years [50]. The treatment resulted in a significant reduction in the overall occurrence of relapses going from 2.2 to 1.0 relapses per patient despite the fact that the post-treatment follow-up was significantly longer when compared with the pre-treatment period [50]. Up to $50 \%$ of the patients remained relapse-free and $76.9 \%$ of patients had no EDSS progression [50]. Lastly, a study that aims at evaluating the safety and efficacy of peginterferon- $\beta$ - $1 \mathrm{a}$ in POMS patients is currently ongoing (ClinicalTrials.gov identifier: NCT03958877).

Studies of much smaller patient numbers have investigated the effectiveness of glatiramer acetate in POMS patients $[51,52]$. Fourteen Italian patients treated with glatiramer acetate were followed for 5.3 years and had a significant reduction in relapse rate from 3.1 before treatment initiation to 0.2 during the follow-up (also termed as the ITEMS study) [51]. A similar reduction was seen in patients who had to switch from glatiramer acetate to other drugs during the follow-up period [51]. A smaller case series (7 patients) also demonstrated a heterogeneous response to glatiramer acetate, with highly active patients not responding to the treatment [52]. As with several probable cases already seen in the AOMS, a case of hepatotoxicity in a pediatric MS patient treated with glatiramer acetate has also been reported [53].

Despite the lack of double-blind studies, two consensus statements (by the International Pediatric MS Study group and a European group) have confirmed the efficacy of injectable DMTs in POMS patients and recommended their use as first-line medications after diagnosis of POMS [54, 55]. The consensus also recognizes the fact that approximately $30 \%$ of the POMS patients would require more aggressive treatment and change to second-line treatment $[54,55]$. The long-term effectiveness of first-line injectable DMTs in POMS was additionally investigated in a group of 97 patients who were followed for an average of 12.5 years. The injectable DMTs resulted in a significant decrease in ARR (from 3.2 to 0.7 ) with EDSS remaining $<4.0$ in $89 \%$ of the patients during the follow-up period. That said, up to $58 \%$ of these cases had to switch to second-line medications during the same timeframe. Changes to second-line therapy are increasingly seen within newer reports that indicate greater use of newer medications as an initial treatment for POMS patients. For example, in a cohort of 1019 patients, up to $17 \%$ of patients had newer medication as the initial therapy choice, including the use of dimethyl fumarate, natalizumab, rituximab, and fingolimod [56]. This increase in newer medications is particularly prevalent among patients that are $\geq 12$ years old [56].

\subsection{Natalizumab}

Currently, there are no randomized, double-blind, interventional trials that investigate the effectiveness and safety of natalizumab (Tysabri ${ }^{\circledR}$ ) in the POMS population. From initial case reports to larger observational studies, this particular drug has been utilized mainly in highly active POMS patients who have failed previous first-line medications such as interferon- $\beta$ and glatiramer acetate [57]. Natalizumab is a humanized monoclonal antibody towards the very late antigen-4 (VLA-4), which blocks the function of vascular 
cell adhesion molecule-1 (VCAM-1) present on the vascular endothelium and impairs the transmigration of lymphocytes through the blood-brain barrier [58]. Firstly, the pharmacokinetic and pharmacodynamic characteristics of natalizumab in pediatric patients are similar to those seen in the adult population [59]. The peak concertation of natalizumab occurs within 1 day after the infusion and is followed by a biphasic decline between the rapid distribution and slow elimination phase [59]. The natalizumab infusion resulted in appropriate saturation of the $\alpha 4$-integrin receptors that remained high through 16 weeks (72.1\% at week 16) [59].

The largest real-world natalizumab study included 101 POMS patients and resulted in a significant reduction of ARR from 2.3 before drug initiation to 0.1 at the last infusion [60]. More than half of the patients $(58 \%)$ remained free of any clinical or MRI activity [60]. Overall, there were no significant occurrences of adverse events and only 19 patients discontinued their medication [60]. The efficacy and safety of natalizumab use in POMS patients were also reviewed in a smaller Portugal-based study called TyPed that retrospectively collected data from 21 patients [61]. In the subset of patients that used natalizumab for 12 months or more, $50 \%$ (6 out of 12) of them experienced a decrease in EDSS scores during the first year and 60\% (6 out of 10) experienced a decrease in EDSS score during the full period of 24 months [61]. A significant reduction in ARR was noted, decreasing from 1.31 before treatment initiation to 0.04 after 24 months while on treatment (only two relapses throughout the entire study) [61]. Only one serious adverse event of pneumonia was recorded and five patients had to discontinue their treatment due to John Cunningham Virus (JCV) seropositivity [61]. Natalizumab has also been used as a first-line therapy in treatment-naïve pediatric MS patients [62]. In a cohort of 20 Italian POMS patients who received on average 42 natalizumab infusions, there were no recorded clinical relapses and only two patients had MRI-based evidence of disease activity [62]. Throughout the 2-year followup time, $80 \%$ of the patients retained their no evidence of disease activity (NEDA-3) status [62]. No treatment discontinuation occurred and at the end of the follow-up period $50 \%$ of the patients were JCV+ [62].

Although it is not approved by the main regulatory agencies such as the US Food and Drug Administration (FDA) and European Medicines Agency (EMA), natalizumab continues to be used in highly active cases and POMS patients that are not responding to first-line medications. For example, a cohort of 32 POMS patients were started on natalizumab due to either having disease breakthrough while on first-line treatment ( $66 \%$ of patients, indication) or being therapy-naïve but having rapidly evolving disease (34\% of the patients) [63]. The treatment resulted in a significant reduction in ARR from 1.66 before treatment to 0.06 at the end of the observational period [63]. Moreover, the treatment reduced the MRI-based activity that was initially present in $93.8 \%$ of the patients to $12.5 \%$ over the follow-up period. Up to $84 \%$ of the patients retained their NEDA-3 status [63]. Given that both the pharmacokinetic/pharmacodynamic (PK/PD) properties and the real-world efficacy/ safety data are similar to those reported in the adult population, natalizumab use in POMS patients could therefore be considered for formal regulatory approval.

\subsection{Fingolimod}

The use of fingolimod (sphingosine-1-phosphate receptor [S1PR] modulator) in POMS patients has been approved by the FDA and EMA based on findings from the PARADIGMS clinical trial [64]. This phase III trial determined the efficacy of $0.5 \mathrm{mg}$ daily fingolimod (Gilenya ${ }^{\circledR}$ ) when compared with $30 \mu \mathrm{g}$ IM interferon $\beta$-1a (Avonex ${ }^{\circledR}$ ) [64]. While fingolimod can exhibit a plethora of immune and nonimmune changes through its binding to various S1P receptors $\left(\mathrm{S}_{1} \mathrm{PR}_{1,3-5}\right)$, the main effect in $\mathrm{MS}$ is attributed to the ability to retain the lymphocytes within the lymphoid tissues and decrease the overall level of lymphocytes in the peripheral circulation. In this double-blind, randomized, active comparator trial, 215 POMS patients, aged 10-17 years, were randomized to either of the treatments and followed for 24 months [64]. Over the follow-up time, the fingolimodtreated patients had a significant $82 \%$ decrease in the ARR (absolute difference of 0.55 ; 95\% CI 0.36-0.74) and a significant $0.53 \%$ decrease in annualized rate of new or newly enlarging T2 lesions (absolute difference of $0.47 ; 95 \% \mathrm{CI}$ 0.36-0.62) [64]. Moreover, a larger portion of fingolimodtreated MS patients were free of in-study relapses $(85.7 \%$ vs $38.8 \%$ ) and had lower average contrast-enhancing lesions per scan (0.44 vs 1.28$)$ when compared with patients treated with IM interferon $\beta$-1a [64]. When compared with data from the comparable adult trial (Efficacy and Safety of Fingolimod in Patients With Relapsing-remitting Multiple Sclerosis With Optional Extension Phase [TRANSFORMS]), the use of fingolimod in the pediatric population resulted in as much as twice the efficacy over IM interferon $\beta-1$ a.

Overall, there were no statistical differences in the amount of any adverse events (88.8\% with fingolimod vs $95.3 \%$ with IM interferon $\beta$-1a), with numerically greater serious adverse events in the fingolimod group (16.8\% vs 6.5\%) [64]. As seen with the adult population, POMS patients treated with fingolimod had more cases of leukopenia, upper respiratory tract infection, and singular occurrences of macular edema, uveitis, and second-degree atrioventricular block, respectively [64]. Fingolimod-treated patients experienced a significant $30 \%$ reduction in absolute lymphocyte count with nadir within the first 2 weeks and remained stable afterward [65]. There was no association between the reduction in absolute lymphocyte count and the occurrence of infections 
[65]. The current guidelines identify two different oral fingolimod doses of either $0.25 \mathrm{mg}$ or $0.5 \mathrm{mg}$ per day based on whether the patient's weight is below or above $40 \mathrm{~kg}$.

A more detailed analysis of the MRI data was additionally performed and published [66]. Fingolimod-treated POMS patients also had a significantly lower occurrence of new T1 hypointense lesions (black holes) compared with IM interferon $\beta-1 \mathrm{a}(62.8 \%$ rate reduction) and a significantly lower annualized rate of brain atrophy $(-0.48 \%$ vs $-0.8 \%)$ [66]. After re-baselining for the first 6 months, the brain atrophy findings did not remain statistically significant [66]. Rebaselining is a common post-hoc analysis that accounts for and corrects for the potential therapy-induced pseudoatrophy [67]. Initiation of effective MS medications could significantly reduce the inflammatory activity and resolve the preexisting brain edema, thus resulting in a spurious increase in brain volume loss [67]. Therefore, the brain atrophy outcomes are commonly measured with the 6-month timepoint acting as the new baseline mark [67]. Both findings suggest better neurodegenerative outcomes with fingolimod treatment when compared with intramuscular interferon $\beta-1 \mathrm{a}$. Lastly, the fingolimod effect on MRI activity remained statistically significant in post-hoc analysis of patients below or above 12 years of age, prior use of DMT, male versus female, and the aforementioned $40-\mathrm{kg}$ weight cut-off [66, 68].

Fingolimod also resulted in continuous numerical improvement in EDSS with a mean change from baseline EDSS to end of the study of -0.23 when compared with worsening of 0.22 in the intramuscular interferon $\beta$-1a group [68]. A greater percentage of fingolimod-treated patients experienced improved EDSS scores (21.9\% vs $12.1 \%$; $p=0.059)$ and significantly fewer patients experienced worsening of EDSS scores (10.5\% vs 20.6\%: $p=0.043$ ) when compared with intramuscular interferon $\beta$-1a [68]. Fingolimod also significantly delayed the time to 3 -month confirmed disability progression by $77.2 \%$ during the 2 years of the trial [68]. When these incremental improvements in outcomes over IM interferon- $\beta$ - 1 a are seen through the economics lens, the use of fingolimod over 2 years provides a significant decrease in overall cost effectiveness to 23,886 Canadian dollars per quality-adjusted life-year (QALY) [69]. An open-label extension following the PARADIGMS population is currently ongoing (NCT01892722).

\subsection{Teriflunomide}

Teriflunomide is a small molecule that creates reversible inhibition of the de novo pyrimidine synthesis, through blockade of the mitochondrial dihydro-orotate dehydrogenase [70]. Since this enzyme is highly expressed in activated and proliferating $\mathrm{T}$ and $\mathrm{B}$ cells, the lack of pyrimidine building blocks would result in interruption of the replication and induce a cytostatic effect [70]. A double-blind, randomized trial has properly investigated the effectiveness of this medication in POMS [71]. This phase III, placebo-controlled study (TERIKIDS trial, NCT02201108) has enrolled up to 166 POMS patients that were randomized to either placebo or the full adult teriflunomide dose of $14 \mathrm{mg}$ (1:2 ratio) [71]. Although teriflunomide treatment resulted in a $34 \%$ reduction in risk of relapse, this primary study outcome was not statistically significant when compared to placebo [72].

The study investigators have listed several reasons that could serve as a potential explanation for the lack of trial significance. One main reason mentioned argues that the placebo group had significantly greater MRI activity during the study, which forced the investigators to switch patients from a double-blind to open-label phase (rescue procedure) [72]. Therefore, a large number of censored placebo patients did not reach the final study outcome, resulting in a much smaller and stable comparison group that unfavored the teriflunomide treatment [72]. In hindsight, a primary trial outcome of time to first relapse would have mitigated this limitation. The 8-week run-in phase for dosage finding in the teriflunomide arm could be considered as another unfavorable trial design that may have allowed more in-trial activity [72]. A post-hoc analysis demonstrated a significant reduction in MRI activity, with a 55\% reduction in new or newly enlarging T2 lesions and a 75\% reduction in gadoliniumenhancing lesions [72]. Similarly, teriflunomide also demonstrated a significant treatment effect on a combined measure of MRI activity and clinical relapses [72]. No teriflunomide effect on reducing brain volume loss was noted [72].

An additional open-label extension study that offered teriflunomide switch after the initial 24 weeks was created. However, early teriflunomide use still was not able to statistically reduce the risk of relapse nor the risk of sustained disability progression. In addition to greater frequency of nasopharyngitis, upper respiratory tract infections, alopecia, and increased blood creatinine levels in the teriflunomide group when compared with placebo, teriflunomide-treated POMS patients also experienced occurrences of pancreatitis [72]. During the open-label extension period, eight patients had to discontinue their treatment, including five cases of increased alanine aminotransferase, one case of peripheral neuropathy, two cases of pancreatitis (one particularly acute and severe case of pancreatitis and pseudo-papilloma), and two cases with pancreatic enzyme elevation [72]. The emergence of pancreatitis in teriflunomide-treated POMS should be addressed with additional guidelines that will outline the steps towards adequate surveillance of these potentially severe adverse events. On 3 July 2021, the European Commission reviewed the TERIKIDS results and approved teriflunomide for treatment of pediatric RRMS patients aged $10-17$ years old. 


\subsection{Dimethyl Fumarate}

Dimethyl fumarate is a fumaric acid ester that has been approved for the treatment of AOMS for almost 10 years. The mechanism of action is mainly mediated by the antioxidant effects of the monomethyl fumarate metabolite and activation of the nuclear factor erythroid-derived 2-related factor (Nrf-2) [73]. Dimethyl fumarate is also associated with anti-inflammatory changes such as a decrease in proinflammatory CD4+ and CD8+ T cells and reduction in adhesion molecules required for lymphocyte migration (intercellular adhesion molecule 1; ICAM-1) [73].

The safety and efficacy of dimethyl fumarate $\left(\right.$ Tecfidera $^{\circledR}$ ) in the POMS population was recently investigated in a small, single-arm, open-label study that consisted of an initial 8-week off-treatment period followed by a 24-week treatment period (FOCUS study; NCT02410200) [74]. An additional 96-week extension termed CONNECTED further followed the same patients that completed the FOCUS study [75]. All patients started the treatment with 1 week of dimethyl fumarate $120 \mathrm{mg}$ twice a day (BID) and later escalated to the full adult dose of $240 \mathrm{mg}$ BID. A total of 15 patients were included for the pre-determined primary endpoint analysis of new or newly enlarging $\mathrm{T} 2$ hyperintense lesions [74]. Treatment with dimethyl fumarate resulted in a significant 3-fold reduction in such lesions when compared with the baseline period. Moreover, the treatment reduced the ARR from 1.5 for the year before entry into the study to 0.8 during the 24-week study period [74].

Over the entire 120-week follow-up through FOCUS and the CONNECTED extension, the ARR remained low at 0.2, an $84.5 \%$ reduction when compared with the year before entry into the study [75]. In the initial 24-week follow-up period, two patients had to discontinue the treatment due to an MS relapse and a case of urticaria, respectively [74]. In terms of serious adverse events, five patients experienced an MS relapse and one patient reported vertigo. Over the extension period, one more case of significant abdominal pain requiring hospitalization was reported [75]. Five patients had absolute lymphocyte count below the lower limit of normal and the total lymphocyte count decreased from baseline to week 24 by $18 \%$ [74]. An additional 3\% decrease was seen through the extension period [75]. A major limitation to this study is the lack of a comparator arm and regressionto-the-mean effect as patients enrolled right after a recent relapse are not necessarily expected to maintain a high level of activity over the treatment period. A larger phase III study that aims to compare the effectiveness of dimethyl fumarate with IM interferon- $\beta-1$ a has been initiated and has an estimated completion date of late 2025 (CONNECT trial, NCT02283853).

\subsection{Anti-CD20 Antibodies}

Rituximab, the first and most widely available anti-CD20 antibody treatment, has been utilized in multiple autoimmune neuroinflammatory diseases including $\mathrm{N}$-methylD-aspartate receptor (NMDAR) disorder, opsoclonus myoclonus ataxia syndrome, neurolupus, NMOSD, MOGAb-associated syndrome, and MS [76]. Binding of the antiCD-20 antibody induces the complement-dependent cytotoxicity and results in depletion of the entire B-cell lineage (except the early progenitor B-cell population and plasma cells that are lacking the CD-20 receptor) [77]. There are only a few case series in the current literature that describe the safety and efficacy of rituximab, including studies from the US Network of Pediatric centers and the Swedish MS registry. Rituximab was one of the most prevalent secondline medications used in the US $(n=56)$, resulting in a $62 \%$ reduction in relapses when compared with first-line injectable therapy (interferon- $\beta$ and glatiramer acetate) [78]. The 14 Swedish POMS patients treated with rituximab had excellent control of disease activity, with no relapses being recorded, stable or decreased EDSS scores, and only one new MRI lesion beyond the first 6 months of treatment [79]. The side effects from rituximab were similar between both case series, with infusion-induced reactions being the most common, followed by rashes and fewer transient increases in liver enzymes [79].

A recent collaborative effort focused on defining and streamlining the use of rituximab in the POMS population, including standardization of the amount and interval of dosing and the duration of the treatment, and suggestions regarding tests performed at drug initiation and at each follow-up visit [80]. Apart from the usual pretreatment with antihistamines, antipyretics, and corticosteroids, rituximab dosing was separated into two schemes that are based on the age and BMI of the child. When compared with the full 1000 -mg dose, children weighing $<40 \mathrm{~kg}$ and younger than 12 years of age should receive only $500 \mathrm{mg}$ separated into two administrations over 2 weeks [80]. An alternative dosing scheme suggests the use of four consecutive doses of $375 \mathrm{mg} / \mathrm{m}^{2}$ that are separated from each other by 1 week. The follow-up re-treatments include a single 1000-mg dose or two $375-\mathrm{mg} / \mathrm{m}^{2}$ doses, respectively [80].

Based on the current success and approval in the AOMS population [77], the humanized anti-CD20 antibody ocrelizumab (Ocrevus ${ }^{\circledR}$ ) is currently being investigated in an openlabel, non-randomized study of 36 POMS patients and aims at determining the safety, pharmacokinetics, and pharmacodynamic effects of the drug (NCT04075266). The depletion and repletion data derived from this study will allow better determination of the proper dosing regimens that should be later investigated in larger regulatory phase III trials. This is particularly important given that pediatric patients may 
exhibit a faster repletion rate when compared with adults [81]. A retrospective medical chart review of pediatric and young AOMS patients treated with ocrelizumab did indicate a greater risk of infusion-related reactions when compared with OPERA I/II findings, with bradycardia being one unique and unexpected reaction [82]. Moreover, the fully humanized anti-CD20 medication ofatumumab (Kespimta ${ }^{\circledR}$ ) is also currently being investigated together with the siponimod (second-generation S1PR modulator, Mayzent ${ }^{\circledR}$ ) in a three-arm, randomized, double-blind, active-controlled (fingolimod) trial that aims to recruit up to 180 POMS patients (NEOS trial, NCT04926818). In order to perform additional post-hoc analyses, the study will also incorporate the addition of at least five patients with BMI $<40 \mathrm{~kg}$ and children aged between $10-12$ years in each study arm.

\subsection{Anti-CD52 Antibodies and Experimental Medications}

In addition to the aforementioned data from the US Network of Pediatric MS centers and IPMSSG, several case reports document stable MS disease and EDSS decrease after two courses of alemtuzumab [83]. As seen in the adult population, POMS patients are also at risk of developing afebrile neutropenia that is likely due to secondary autoimmune etiology [83]. Alemtuzumab (a humanized monoclonal antiCD52 antibody that results in the depletion of T and B cells) can also be utilized as a follow-up medication in highly active POMS patients that discontinue their natalizumab protocol due to high JCV index [84]. Five such young adult/ adolescent patients retained their NEDA-3 status throughout the natalizumab treatment and 2 years after their first alemtuzumab course. Moreover, three out of five patients had improvement in their EDSS score [84]. The safety and efficacy of alemtuzumab in POMS patients are also currently being investigated in an open-label, non-randomized study that aims at enrolling 50 pediatric RRMS patients with documented disease activity on previous DMT (LemKids trial, NCT03368664).

Although still not approved for use in the AOMS population, a vaccine that can induce immunity towards encephalitogenic $\mathrm{T}$ cells that express specific T-cell receptors (TCR) is currently being tested in 12 POMS patients (NCT02200718). This intervention aims at reducing the MBP-specific T cells, which would prevent MS-specific inflammation [85]. Multiple and fairly old case series have also explored the use of cyclophosphamide treatment in POMS patients [86].

Lastly, the European Society for Blood and Marrow Transplantation (EBMT) has reported on the use of autologous hematopoietic stem cell transplantation (aHSCT) in 21 POMS patients [87]. The median age of the patients at the time of transplant was 16 years old, with age of diagnosis at 13 years old and a median of three relapses in the year before the procedure (up to 12 relapses in one patient) [87]. Before the conditioning protocols (BEAM [BCNU, etoposide, cytosine-arabinoside, and melphalan], cyclophosphamide or ATG protocols), the peripheral hematopoietic stem cells were mobilized with filgrastim and cyclophosphamide [87]. The ARR after the aHSCT procedure was reduced to 0.022 (where only 2 patients experienced post-aHSCT relapse) [87]. Up to 16 patients had improvement in their EDSS scores with a median improvement of 2.0 EDSS points [87]. Over the follow-up period of 3 years, one patient was hospitalized due to Pseudomonas aeruginosa sepsis, two cases had culture-confirmed bacteremia, and 12 patients had a culture-negative fever [87]. Reactivations in cytomegalovirus (CMV) and EBV were treated with ganciclovir and foscarnet, respectively [87]. Given that the use of aHSCT as a treatment for aggressive MS is of increasing interest, future larger studies would provide more data regarding the safety and efficacy of this procedure.

\section{Conclusion}

Albeit rare, diagnosis of POMS has significant long-term implications for these children that could affect their physical and cognitive development and influence their life-long quality of life. Since these outcomes are commonly seen despite intervention with first-line DMTs, a newer and more efficacious set of interventions is highly warranted. Therefore, early and correct diagnosis, coupled with early initiation of an appropriate DMT is of utmost importance. The recent approval of several oral medications such as fingolimod and teriflunomide and greater real-world use of more potent medications is a step towards this direction, but more data remains to be pursued.

A recent IPMSSG survey of medical professionals did outline the current tendency to move away from the traditional escalation therapy and start favoring early highly effective treatment choices for their POMS patients [88]. Almost half of the responders mentioned the use of highefficacy drugs as their first DMT and only one neurologist did not consider induction therapy as an option. The Coronavirus disease 2019 (COVID-19) pandemic has not deterred the use of high-efficacy treatments either, with up to $84 \%$ of respondents choosing to use anti-CD20 medications or oral DMTs, despite the pandemic [88]. Moreover, a greater percentage of respondents $(61 \%)$ would not consider postponing anti-CD20 doses [88]. Lastly, only up to $31 \%$ of the respondents indicated that they have changed their DMT prescribing practice (with emphasis on the early pandemic period) [88].

The discrepancies in drug availability between AOMS and POMS patients can be attributed to several methodological 
and practical limitations that generally lead to a delay in approval by 8 years. Regrettably, these discrepancies still exist despite the large body of evidence that suggests a comparable safety profile between POMS and AOMS. A recent statement by the International Pediatric MS Study group outlined multiple challenges and opportunities that could improve the clinical trial designs and drug approval [89].

Firstly, the prevalence of POMS is significantly lower when compared with AOMS, and therefore the recruitment for larger phase III trials is commonly laborious and long. Secondly, the use of placebo-controlled studies in view of AOMS data is arguably unethical and generally not justified. Thirdly, most recommendations regarding the safety of DMT use in POMS patients are commonly derived from longer observational studies and the short 2-year trials are insufficient to properly capture the occurrence of adverse events [89]. Therefore, all POMS trials should mandate openlabel extension studies. Unless the mechanism of action is expected to be different between the adult and pediatric patients, the drug approval should only be based on PK/PD studies to determine the optimal pediatric doses [89].

Alternately, future phase III trials in AOMS patients that investigate drugs with previously demonstrated strong safety profiles could consider the inclusion of teenagers. Lastly, and if the data from the PK/PD study seems insufficient for drug approval, the drug should be investigated in a smaller controlled trial where the clinical-based outcomes should be replaced by MRI endpoints [89]. A recent opinion paper also emphasized the current limitations regarding the use of randomized clinical trials in POMS and urged the regulatory agencies to reconsider the process of assessment and approval of new POMS-based medications [90].

\section{Declarations}

Funding The Authors have not received any direct funding that is associated with this manuscript.

Conflicts of interest/Competing interests Dejan Jakimovski, Samreen Awan, Svetlana P. Eckert, and Osman Farooq have nothing to disclose. Dr Bianca Weinstock-Guttman has participated in speaker's bureaus and/or served as a consultant for Biogen, EMD Serono, Novartis, Genentech, Celgene/Bristol Meyers Squibb, Sanofi Genzyme, Bayer, Janssen, and Horizon. Dr Weinstock-Guttman also has received grant/ research support from the agencies listed in the previous sentence. She serves on the editorial board for BMJ Neurology, Children, CNS Drugs, MS International, and Frontiers Epidemiology.

Ethics approval Not applicable.

Availability of data and material Not applicable.

Code availability Not applicable.

Consent to participate Not applicable.

Consent for publication Not applicable.
Authors contributions All authors have contributed to drafting and reviewing of the manuscript. All authors approved the final version of the manuscript and agree to be accountable for the work presented in the manuscript.

\section{References}

1. Belman AL, Krupp LB, Olsen CS, Rose JW, Aaen G, Benson $\mathrm{L}$, et al. Characteristics of children and adolescents with multiple sclerosis. Pediatrics. 2016. https://doi.org/10.1542/peds. 2016-0120.

2. Renoux C, Vukusic S, Mikaeloff Y, Edan G, Clanet M, Dubois B, et al. Natural history of multiple sclerosis with childhood onset. N Engl J Med. 2007;356(25):2603-13. https://doi.org/10.1056/ NEJMoa067597.

3. Chitnis T, Aaen G, Belman A, Benson L, Gorman M, Goyal MS, et al. Improved relapse recovery in paediatric compared to adult multiple sclerosis. Brain. 2020;143(9):2733-41. https://doi.org/ 10.1093/brain/awaa199.

4. Bartels F, Nobis K, Cooper G, Wendel E, Cleaveland R, BajerKornek B, et al. Childhood multiple sclerosis is associated with reduced brain volumes at first clinical presentation and brain growth failure. Mult Scler. 2019;25(7):927-36. https://doi.org/ 10.1177/1352458519829698.

5. Wallach AI, Waltz M, Casper TC, Aaen G, Belman A, Benson $\mathrm{L}$, et al. Cognitive processing speed in pediatric-onset multiple sclerosis: Baseline characteristics of impairment and prediction of decline. Mult Scler. 2020;26(14):1938-47. https://doi.org/10. $1177 / 1352458519891984$.

6. McKay KA, Friberg E, Razaz N, Alexanderson K, Hillert J. Longterm socioeconomic outcomes associated with pediatric-onset multiple sclerosis. JAMA Neurol. 2021;78(4):478-82. https:// doi.org/10.1001/jamaneurol.2020.5520.

7. Baroncini D, Simone M, Iaffaldano P, Brescia Morra V, Lanzillo R, Filippi M, et al. Risk of persistent disability in patients with pediatric-onset multiple sclerosis. JAMA Neurol. 2021;78(6):72635. https://doi.org/10.1001/jamaneurol.2021.1008.

8. Jeong A, Oleske DM, Holman J. Epidemiology of pediatric-onset multiple sclerosis: a systematic review of the literature. J Child Neurol. 2019;34(12):705-12. https://doi.org/10.1177/0883073819 845827.

9. Yan K, Balijepalli C, Desai K, Gullapalli L, Druyts E. Epidemiology of pediatric multiple sclerosis: a systematic literature review and meta-analysis. Mult Scler Relat Disord. 2020;44: 102260. https://doi.org/10.1016/j.msard.2020.102260.

10. Marrie RA, O'Mahony J, Maxwell C, Ling V, Yeh EA, Arnold DL, et al. Incidence and prevalence of MS in children: a populationbased study in Ontario, Canada. Neurology. 2018;91(17):e157990. https://doi.org/10.1212/WNL.0000000000006395.

11. Boesen MS, Jensen PEH, Born AP, Magyari M, Nilsson AC, Hoei-Hansen $\mathrm{C}$, et al. Incidence of pediatric neuromyelitis optica spectrum disorder and myelin oligodendrocyte glycoprotein antibody-associated disease in Denmark 20082018: a nationwide, population-based cohort study. Mult Scler Relat Disord. 2019;33:162-7. https://doi.org/10.1016/j.msard.2019.06.002.

12. Sepulveda M, Aldea M, Escudero D, Llufriu S, Arrambide G, Otero-Romero S, et al. Epidemiology of NMOSD in Catalonia: Influence of the new 2015 criteria in incidence and prevalence estimates. Mult Scler. 2018;24(14):1843-51. https://doi.org/10. $1177 / 1352458517735191$.

13. Yamaguchi Y, Torisu H, Kira R, Ishizaki Y, Sakai Y, Sanefuji M, et al. A nationwide survey of pediatric acquired demyelinating 
syndromes in Japan. Neurology. 2016;87(19):2006-15. https:// doi.org/10.1212/WNL.0000000000003318.

14. Fadda G, Armangue T, Hacohen Y, Chitnis T, Banwell B. Paediatric multiple sclerosis and antibody-associated demyelination: clinical, imaging, and biological considerations for diagnosis and care. Lancet Neurol. 2021;20(2):136-49. https://doi.org/10.1016/ S1474-4422(20)30432-4.

15. Fadda G, Brown RA, Longoni G, Castro DA, O’Mahony J, Verhey $\mathrm{LH}$, et al. MRI and laboratory features and the performance of international criteria in the diagnosis of multiple sclerosis in children and adolescents: a prospective cohort study. Lancet Child Adolesc Health. 2018;2(3):191-204. https://doi.org/10.1016/ S2352-4642(18)30026-9.

16. Verhey LH, Branson HM, Shroff MM, Callen DJ, Sled JG, Narayanan S, et al. MRI parameters for prediction of multiple sclerosis diagnosis in children with acute CNS demyelination: a prospective national cohort study. Lancet Neurol. 2011;10(12):1065-73. https://doi.org/10.1016/S1474-4422(11)70250-2.

17. Wingerchuk DM, Banwell B, Bennett JL, Cabre P, Carroll W, Chitnis T, et al. International consensus diagnostic criteria for neuromyelitis optica spectrum disorders. Neurology. 2015;85(2):17789. https://doi.org/10.1212/WNL.0000000000001729.

18. Hacohen Y, Mankad K, Chong WK, Barkhof F, Vincent A, Lim $\mathrm{M}$, et al. Diagnostic algorithm for relapsing acquired demyelinating syndromes in children. Neurology. 2017;89(3):269-78. https:// doi.org/10.1212/WNL.0000000000004117.

19 Bruijstens AL, Lechner C, Flet-Berliac L, Deiva K, Neuteboom RF, Hemingway C, et al. E.U. paediatric MOG consortium consensus: part $1-$ classification of clinical phenotypes of paediatric myelin oligodendrocyte glycoprotein antibody-associated disorders. Eur J Paediatr Neurol. 2020;29:2-13. https://doi.org/10. 1016/j.ejpn.2020.10.006.

20. Baumann M, Bartels F, Finke C, Adamsbaum C, Hacohen Y, Rostasy K, et al. E.U. paediatric MOG consortium consensus: Part 2-Neuroimaging features of paediatric myelin oligodendrocyte glycoprotein antibody-associated disorders. Eur J Paediatr Neurol. 2020;29:14-21. https://doi.org/10.1016/j.ejpn.2020.10.002.

21 Armangue T, Capobianco M, de Chalus A, Laetitia G, Deiva K, consortium EUpM. E.U. paediatric MOG consortium consensus: part 3-biomarkers of paediatric myelin oligodendrocyte glycoprotein antibody-associated disorders. Eur J Paediatr Neurol. 2020;29:22-31. https://doi.org/10.1016/j.ejpn.2020.11.001.

22 Bruijstens AL, Breu M, Wendel EM, Wassmer E, Lim M, Neuteboom RF, et al. EU paediatric MOG consortium consensus: part 4-outcome of paediatric myelin oligodendrocyte glycoprotein antibody-associated disorders. Eur J Paediatr Neurol. 2020;29:3240. https://doi.org/10.1016/j.ejpn.2020.10.007.

23 Bruijstens AL, Wendel EM, Lechner C, Bartels F, Finke C, Breu M, et al. E.U .paediatric MOG consortium consensus: part 5treatment of paediatric myelin oligodendrocyte glycoprotein antibody-associated disorders. Eur J Paediatr Neurol. 2020;29:41-53. https://doi.org/10.1016/j.ejpn.2020.10.005.

24. Santoro JD, Waltz M, Aaen G, Belman A, Benson L, Gorman $\mathrm{M}$, et al. Pediatric multiple sclerosis severity score in a large US cohort. Neurology. 2020;95(13):e1844-53. https://doi.org/10. 1212/WNL.0000000000010414.

25. McKay KA, Hillert J, Manouchehrinia A. Long-term disability progression of pediatric-onset multiple sclerosis. Neurology. 2019;92(24):e2764-73. https://doi.org/10.1212/WNL.00000 00000007647

26. Mikaeloff Y, Caridade G, Assi S, Suissa S, Tardieu M. Prognostic factors for early severity in a childhood multiple sclerosis cohort. Pediatrics. 2006;118(3):1133-9. https://doi.org/10.1542/peds. 2006-0655

27. Akhtar S, Alroughani R, Ahmed SF, Al-Hashel JY. Prognostic indicators of secondary progression in a paediatric-onset multiple sclerosis cohort in Kuwait. Mult Scler. 2016;22(8):1086-93. https://doi.org/10.1177/1352458515608960.

28. De Meo E, Bonacchi R, Moiola L, Colombo B, Sangalli F, Zanetta $\mathrm{C}$, et al. Early predictors of 9-year disability in pediatric multiple sclerosis. Ann Neurol. 2021;89(5):1011-22. https://doi.org/10. 1002/ana.26052.

29. Kopp TI, Blinkenberg M, Chalmer TA, Petersen T, Ravnborg $\mathrm{MH}$, Soelberg Sorensen P, et al. Predictors of treatment outcome in patients with paediatric onset multiple sclerosis. Mult Scler. 2020;26(8):964-75. https://doi.org/10.1177/1352458519846100.

30. Kopp TI, Blinkenberg M, Petersen T, Sorensen PS, Magyari M. Long term effect of delayed treatment on disability in patients with paediatric onset multiple sclerosis: a prospective Danish cohort study. Mult Scler Relat Disord. 2020;40: 101956. https:// doi.org/10.1016/j.msard.2020.101956.

31. McKay KA, Manouchehrinia A, Berrigan L, Fisk JD, Olsson T, Hillert J. Long-term cognitive outcomes in patients with pediatric-onset vs adult-onset multiple sclerosis. JAMA Neurol. 2019;76(9):1028-34. https://doi.org/10.1001/jamaneurol.2019. 1546.

32. De Meo E, Meani A, Moiola L, Ghezzi A, Veggiotti P, Filippi M, et al. Dynamic gray matter volume changes in pediatric multiple sclerosis: a 3.5 year MRI study. Neurology. 2019;92(15):e170923. https://doi.org/10.1212/WNL.0000000000007267.

33. Langer-Gould A, Brara SM, Beaber BE, Koebnick C. Childhood obesity and risk of pediatric multiple sclerosis and clinically isolated syndrome. Neurology. 2013;80(6):548-52. https://doi.org/ 10.1212/WNL.0b013e31828154f3.

34. Pakpoor J, Disanto G, Gerber JE, Dobson R, Meier UC, Giovannoni $\mathrm{G}$, et al. The risk of developing multiple sclerosis in individuals seronegative for Epstein-Barr virus: a meta-analysis. Mult Scler. 2013;19(2):162-6. https://doi.org/10.1177/1352458512 449682.

35. Mowry EM, Krupp LB, Milazzo M, Chabas D, Strober JB, Belman AL, et al. Vitamin D status is associated with relapse rate in pediatric-onset multiple sclerosis. Ann Neurol. 2010;67(5):61824. https://doi.org/10.1002/ana.21972.

36. Gianfrancesco MA, Stridh P, Shao X, Rhead B, Graves JS, Chitnis $\mathrm{T}$, et al. Genetic risk factors for pediatric-onset multiple sclerosis. Mult Scler. 2018;24(14):1825-34. https://doi.org/10.1177/13524 58517733551.

37. Gianfrancesco MA, Stridh P, Rhead B, Shao X, Xu E, Graves JS, et al. Evidence for a causal relationship between low vitamin D, high BMI, and pediatric-onset MS. Neurology. 2017;88(17):16239. https://doi.org/10.1212/WNL.0000000000003849.

38. Jakimovski D, Guan Y, Ramanathan M, Weinstock-Guttman B, Zivadinov R. Lifestyle-based modifiable risk factors in multiple sclerosis: review of experimental and clinical findings. Neurodegener Dis Manag. 2019;9(3):149-72. https://doi.org/10.2217/ nmt-2018-0046.

39 Jakimovski D, Kolb C, Ramanathan M, Zivadinov R, WeinstockGuttman B. Interferon beta for multiple sclerosis. Cold Spring Harb Perspect Med. 2018. https://doi.org/10.1101/cshperspect. a032003.

40. Weinstock-Guttman B, Nair KV, Glajch JL, Ganguly TC, Kantor D. Two decades of glatiramer acetate: from initial discovery to the current development of generics. J Neurol Sci. 2017;376:255-9. https://doi.org/10.1016/j.jns.2017.03.030.

41. Pohl D, Rostasy K, Gartner J, Hanefeld F. Treatment of early onset multiple sclerosis with subcutaneous interferon beta-1a. Neurology. 2005;64(5):888-90. https://doi.org/10.1212/01.WNL.00001 53570.33845.6A.

42. Banwell B, Reder AT, Krupp L, Tenembaum S, Eraksoy M, Alexey B, et al. Safety and tolerability of interferon beta- $1 \mathrm{~b}$ in pediatric multiple sclerosis. Neurology. 2006;66(4):472-6. https:// doi.org/10.1212/01.wnl.0000198257.52512.1a. 
43. Ghezzi A, Amato MP, Capobianco M, Gallo P, Marrosu MG, Martinelli V, et al. Treatment of early-onset multiple sclerosis with intramuscular interferonbeta-1a: long-term results. Neurol Sci. 2007;28(3):127-32. https://doi.org/10.1007/s10072-007-0804-2.

44. Kappos L, Polman CH, Freedman MS, Edan G, Hartung HP, Miller DH, et al. Treatment with interferon beta-1b delays conversion to clinically definite and McDonald MS in patients with clinically isolated syndromes. Neurology. 2006;67(7):1242-9. https://doi.org/10.1212/01.wnl.0000237641.33768.8d.

45. Comi G, Filippi M, Barkhof F, Durelli L, Edan G, Fernandez $\mathrm{O}$, et al. Effect of early interferon treatment on conversion to definite multiple sclerosis: a randomised study. Lancet. 2001;357(9268):1576-82. https://doi.org/10.1016/s01406736(00)04725-5.

46 Jacobs LD, Beck RW, Simon JH, Kinkel RP, Brownscheidle CM, Murray TJ, et al. Intramuscular interferon beta-1a therapy initiated during a first demyelinating event in multiple sclerosis. CHAMPS Study Group. N Engl J Med. 2000;343(13):898-904. https://doi. org/10.1056/NEJM200009283431301.

47 Mikaeloff Y, Caridade G, Tardieu M, Suissa S, Society KsgotFN. Effectiveness of early beta interferon on the first attack after confirmed multiple sclerosis: a comparative cohort study. Eur J Paediatr Neurol. 2008;12(3):205-9. https://doi.org/10.1016/j.ejpn.2007. 08.001 .

48. Tenembaum SN, Banwell B, Pohl D, Krupp LB, Boyko A, Meinel $\mathrm{M}$, et al. Subcutaneous interferon Beta-1a in pediatric multiple sclerosis: a retrospective study. J Child Neurol. 2013;28(7):84956. https://doi.org/10.1177/0883073813488828.

49. Krupp LB, Pohl D, Ghezzi A, Boyko A, Tenembaum S, Chen $\mathrm{L}$, et al. Subcutaneous interferon beta-1a in pediatric patients with multiple sclerosis: Regional differences in clinical features, disease management, and treatment outcomes in an international retrospective study. J Neurol Sci. 2016;363:33-8. https://doi.org/ 10.1016/j.jns.2016.01.023.

50. Gartner J, Bruck W, Weddige A, Hummel H, Norenberg C, Bugge $\mathrm{JP}$, et al. Interferon beta- $1 \mathrm{~b}$ in treatment-naive paediatric patients with relapsing-remitting multiple sclerosis: Two-year results from the BETAPAEDIC study. Mult Scler J Exp Transl Clin. 2017;3(4):2055217317747623. https://doi.org/10.1177/20552 17317747623 .

51. Ghezzi A, Amato MP, Annovazzi P, Capobianco M, Gallo P, La Mantia L, et al. Long-term results of immunomodulatory treatment in children and adolescents with multiple sclerosis: the Italian experience. Neurol Sci. 2009;30(3):193-9. https://doi.org/10. 1007/s10072-009-0083-1.

52. Kornek B, Bernert G, Balassy C, Geldner J, Prayer D, Feucht M. Glatiramer acetate treatment in patients with childhood and juvenile onset multiple sclerosis. Neuropediatrics. 2003;34(3):120-6. https://doi.org/10.1055/s-2003-41274.

53. Makhani N, Ngan BY, Kamath BM, Yeh EA. Glatiramer acetateinduced acute hepatotoxicity in an adolescent with MS. Neurology. 2013;81(9):850-2. https://doi.org/10.1212/WNL.0b013 e3182a2cc4a.

54. Chitnis T, Tenembaum S, Banwell B, Krupp L, Pohl D, Rostasy K, et al. Consensus statement: evaluation of new and existing therapeutics for pediatric multiple sclerosis. Mult Scler. 2012;18(1):116-27. https://doi.org/10.1177/1352458511430704.

55. Ghezzi A, Banwell B, Boyko A, Amato MP, Anlar B, Blinkenberg M, et al. The management of multiple sclerosis in children: a European view. Mult Scler. 2010;16(10):1258-67. https://doi. org/10.1177/1352458510375568.

56. Krysko KM, Graves J, Rensel M, Weinstock-Guttman B, Aaen G, Benson $\mathrm{L}$, et al. Use of newer disease-modifying therapies in pediatric multiple sclerosis in the US. Neurology. 2018;91(19):e177887. https://doi.org/10.1212/WNL.0000000000006471.
57. Yeh EA, Weinstock-Guttman B. Natalizumab in pediatric multiple sclerosis patients. Ther Adv Neurol Disord. 2010;3(5):293-9. https://doi.org/10.1177/1756285610381526.

58. Gandhi S, Jakimovski D, Ahmed R, Hojnacki D, Kolb C, Weinstock-Guttman B, et al. Use of natalizumab in multiple sclerosis: current perspectives. Expert Opin Biol Ther. 2016;16(9):1151-62. https://doi.org/10.1080/14712598.2016.1213810.

59. Ghezzi A, Comi G, Grimaldi LM, Moiola L, Pozzilli C, Fantaccini $\mathrm{S}$, et al. Pharmacokinetics and pharmacodynamics of natalizumab in pediatric patients with RRMS. Neurol Neuroimmunol Neuroinflamm. 2019;6(5): e591. https://doi.org/10.1212/NXI.0000000000 000591.

60. Ghezzi A, Moiola L, Pozzilli C, Brescia-Morra V, Gallo P, Grimaldi LM, et al. Natalizumab in the pediatric MS population: results of the Italian registry. BMC Neurol. 2015;15:174. https:// doi.org/10.1186/s12883-015-0433-y.

61. Palavra F, Figueiroa S, Correia AS, Tapadinhas F, Cerqueira J, Guerreiro RP, et al. TyPed study: Natalizumab for the treatment of pediatric-onset multiple sclerosis in Portugal. Mult Scler Relat Disord. 2021;51: 102865. https://doi.org/10.1016/j.msard.2021. 102865.

62. Margoni M, Rinaldi F, Riccardi A, Franciotta S, Perini P, Gallo P. No evidence of disease activity including cognition (NEDA-3 plus) in naive pediatric multiple sclerosis patients treated with natalizumab. J Neurol. 2020;267(1):100-5. https://doi.org/10. 1007/s00415-019-09554-z.

63. Alroughani R, Ahmed SF, Behbehani R, Al-Hashel J. The use of natalizumab in pediatric patients with active relapsing multiple sclerosis: a prospective study. Pediatr Neurol. 2017;70:56-60. https://doi.org/10.1016/j.pediatrneurol.2017.01.017.

64. Chitnis T, Arnold DL, Banwell B, Bruck W, Ghezzi A, Giovannoni $\mathrm{G}$, et al. Trial of fingolimod versus interferon beta-1a in pediatric multiple sclerosis. N Engl J Med. 2018;379(11):1017-27. https://doi.org/10.1056/NEJMoa1800149.

65. Chitnis T, Banwell B, Krupp L, Arnold DL, Bar-Or A, Bruck W, et al. Temporal profile of lymphocyte counts and relationship with infections with fingolimod therapy in paediatric patients with multiple sclerosis: Results from the PARADIGMS study. Mult Scler. 2021;27(6):922-32. https://doi.org/10.1177/1352458520936934.

66. Arnold DL, Banwell B, Bar-Or A, Ghezzi A, Greenberg BM, Waubant E, et al. Effect of fingolimod on MRI outcomes in patients with paediatric-onset multiple sclerosis: results from the phase 3 PARADIGMS study. J Neurol Neurosurg Psychiatry. 2020;91(5):483-92. https://doi.org/10.1136/jnnp-2019-322138.

67 Zivadinov R, Jakimovski D, Gandhi S, Ahmed R, Dwyer MG, Horakova $\mathrm{D}$, et al. Clinical relevance of brain atrophy assessment in multiple sclerosis. Implications for its use in a clinical routine. Expert Rev Neurother. 2016;16(7):777-93. https://doi.org/10. 1080/14737175.2016.1181543.

68. Deiva K, Huppke P, Banwell B, Chitnis T, Gartner J, Krupp L, et al. Consistent control of disease activity with fingolimod versus IFN beta-1a in paediatric-onset multiple sclerosis: further insights from PARADIGMS. J Neurol Neurosurg Psychiatry. 2020;91(1):58-66. https://doi.org/10.1136/jnnp-2019-321124.

69. Nakhaipour HR, Vudumula U, Khurana V, Sebire G, Mah JK, Pohl $\mathrm{D}$, et al. Cost-effectiveness of fingolimod versus interferon-beta1a for the treatment of pediatric-onset multiple sclerosis in Canada. $\mathbf{J}$ Med Econ. 2020;23(12):1525-33. https://doi.org/10.1080/13696 998.2020 .1840138$.

70. Bar-Or A, Pachner A, Menguy-Vacheron F, Kaplan J, Wiendl H. Teriflunomide and its mechanism of action in multiple sclerosis. Drugs. 2014;74(6):659-74. https://doi.org/10.1007/ s40265-014-0212-x.

71 Chitnis T, Tardieu M, Banwell B, Gücüyener K, Deiva K, Skripchenko $\mathrm{N}$, et al. Evaluation of teriflunomide in children and adolescents with relapsing ms: terikids phase 3 study design, 
enrollment update, and baseline data (P4.354). Neurology. 2018;90(15 Supplement):P4.354.

72. Chitnis T, Banwell B, Kappos L, Arnold DL, Gucuyener K, Deiva K, et al. Safety and efficacy of teriflunomide in paediatric multiple sclerosis (TERIKIDS): a multicentre, double-blind, phase 3, randomised, placebo-controlled trial. Lancet Neurol. 2021;20(12):1001-11. https://doi.org/10.1016/S1474-4422(21) 00364-1.

73. Ozel O, Vaughn CB, Eckert SP, Jakimovski D, Lizarraga AA, Weinstock-Guttman B. Dimethyl Fumarate in the treatment of relapsing-remitting multiple sclerosis: patient reported outcomes and perspectives. Patient Relat Outcome Meas. 2019;10:373-84. https://doi.org/10.2147/PROM.S168095.

74. Alroughani R, Das R, Penner N, Pultz J, Taylor C, Eraly S. Safety and efficacy of delayed-release dimethyl fumarate in pediatric patients with relapsing multiple sclerosis (FOCUS). Pediatr Neurol. 2018;83:19-24. https://doi.org/10.1016/j.pediatrneurol.2018. 03.007 .

75. Alroughani R, Huppke P, Mazurkiewicz-Beldzinska M, Blaschek A, Valis M, Aaen G, et al. Delayed-release dimethyl fumarate safety and efficacy in pediatric patients with relapsing-remitting multiple sclerosis. Front Neurol. 2020;11: 606418. https://doi.org/ 10.3389/fneur.2020.606418.

76. Dale RC, Brilot F, Duffy LV, Twilt M, Waldman AT, Narula S, et al. Utility and safety of rituximab in pediatric autoimmune and inflammatory CNS disease. Neurology. 2014;83(2):142-50. https://doi.org/10.1212/WNL.0000000000000570.

77. Jakimovski D, Weinstock-Guttman B, Ramanathan M, Kolb C, Hojnacki D, Minagar A, et al. Ocrelizumab: a B-cell depleting therapy for multiple sclerosis. Expert Opin Biol Ther. 2017;17(9):1163-72. https://doi.org/10.1080/14712598.2017. 1347632 .

78. Krysko KM, Graves JS, Rensel M, Weinstock-Guttman B, Rutatangwa A, Aaen G, et al. Real-world effectiveness of initial disease-modifying therapies in pediatric multiple sclerosis. Ann Neurol. 2020;88(1):42-55. https://doi.org/10.1002/ana.25737.

79. Salzer J, Lycke J, Wickstrom R, Naver H, Piehl F, Svenningsson A. Rituximab in paediatric onset multiple sclerosis: a case series. J Neurol. 2016;263(2):322-6. https://doi.org/10.1007/ s00415-015-7979-x

80 Ghezzi A, Banwell B, Bar-Or A, Chitnis T, Dale RC, Gorman $\mathrm{M}$, et al. Rituximab in patients with pediatric multiple sclerosis and other demyelinating disorders of the CNS: Practical considerations. Mult Scler. 2020. https://doi.org/10.1177/1352458520 932798.

81. Nosadini M, Alper G, Riney CJ, Benson LA, Mohammad SS, Ramanathan $\mathrm{S}$, et al. Rituximab monitoring and redosing in pediatric neuromyelitis optica spectrum disorder. Neurol Neuroimmunol Neuroinflamm. 2016;3(1): e188. https://doi.org/10.1212/ NXI.0000000000000188.

82. Goldschmidt C, Rensel M, Mahajan K, Amin M. A retrospective chart review of the safety and tolerability in ocrelizumab in pediatric and young-adult onset multiple sclerosis (4568). Neurology. 2020;94(15 Supplement):4568.

83. Jure Hunt D, Traboulsee A. Short-term outcomes of pediatric multiple sclerosis patients treated with alemtuzumab at a Canadian University multiple sclerosis clinic. Mult Scler J Exp Transl Clin. 2020;6(2):2055217320926613. https://doi.org/10.1177/20552 17320926613 .

84. Margoni M, Rinaldi F, Miante S, Franciotta S, Perini P, Gallo P. Alemtuzumab following natalizumab in highly active paediatric-onset multiple sclerosis. Mult Scler J Exp Transl Clin. 2019;5(3):2055217319875471. https://doi.org/10.1177/20552 17319875471.

85 Jakimovski D, Weinstock-Guttman B, Ramanathan M, Dwyer MG, Zivadinov R. Infections, vaccines and autoimmunity: a multiple sclerosis perspective. Vaccines. 2020. https://doi.org/ 10.3390/vaccines 8010050 .

86. Gonsette RE, Demonty L, Delmotte P. Intensive immunosuppression with cyclophosphamide in multiple sclerosis. Follow up of 110 patients for 2-6 years. J Neurol. 1977;214(3):173-81. https:// doi.org/10.1007/BF00316148.

87. Burman J, Kirgizov K, Carlson K, Badoglio M, Mancardi GL, De Luca G, et al. Autologous hematopoietic stem cell transplantation for pediatric multiple sclerosis: a registry-based study of the Autoimmune Diseases Working Party (ADWP) and Pediatric Diseases Working Party (PDWP) of the European Society for Blood and Marrow Transplantation (EBMT). Bone Marrow Transplant. 2017;52(8):1133-7. https://doi.org/10.1038/bmt.2017.40.

88. Sandesjo F, Wassmer E, Deiva K, Amato MP, Chitnis T, Hemingway $\mathrm{C}$, et al. Current international trends in the treatment of multiple sclerosis in children-Impact of the COVID-19 pandemic. Mult Scler Relat Disord. 2021;56: 103277. https://doi.org/10.1016/j. msard.2021.103277.

89. Waubant E, Banwell B, Wassmer E, Sormani MP, Amato MP, Hintzen R, et al. Clinical trials of disease-modifying agents in pediatric MS: Opportunities, challenges, and recommendations from the IPMSSG. Neurology. 2019;92(22):e2538-49. https://doi. org/10.1212/WNL.0000000000007572.

90. Ghezzi A, Amato MP, Edan G, Hartung HP, Havrdova EK, Kappos L, et al. The introduction of new medications in pediatric multiple sclerosis: open issues and challenges. Mult Scler. 2021;27(3):479-82. https://doi.org/10.1177/1352458520930620. 